\title{
Numerical solution and parameter estimation for uncertain SIR model with application to COVID-19
}

\author{
Xiaowei Chen ${ }^{1}\left[\right.$ ] Jing $\mathrm{Li}^{2} \cdot$ Chen Xiao ${ }^{1} \cdot$ Peilin Yang ${ }^{1}$ \\ Accepted: 7 September 2020 / Published online: 17 September 2020 \\ (c) Springer Science+Business Media, LLC, part of Springer Nature 2020
}

\begin{abstract}
Developing algorithms for solving high-dimensional uncertain differential equations has been an exceedingly difficult task. This paper presents an $\alpha$-path-based approach that can handle the proposed high-dimensional uncertain SIR model. We apply the $\alpha$ path-based approach to calculating the uncertainty distributions and related expected values of the solutions. Furthermore, we employ the method of moments to estimate parameters and design a numerical algorithm to solve them. This model is applied to describing the development trend of COVID-19 using infected and recovered data of Hubei province. The results indicate that lockdown policy achieves almost $100 \%$ efficiency after February 13, 2020, which is consistent with the existing literatures. The high-dimensional $\alpha$-path-based approach opens up new possibilities in solving high-dimensional uncertain differential equations and new applications.
\end{abstract}

Keywords Uncertainty theory · Uncertain differential equation · SIR model · COVID-19

\section{Introduction}

The World Health Organization (WHO) defines a pandemic as the worldwide spread of a new disease. Since December 2019, the COVID-19 causes infection of over 10

Jing Li
lijingnk@ nankai.edu.cn
Xiaowei Chen
chenx@ nankai.edu.cn
Chen Xiao
xc@mail.nankai.edu.cn
Peilin Yang
yang.peilinc@gmail.com
1 School of Finance, Nankai University, Tianjin 300350, China
$2 \quad$ School of Mathematical Sciences, Nankai University, Tianjin 300071, China 
million people and over $500 \mathrm{~K}$ deaths by Jun 30, 2020. The widespread epidemics have a profound historical impact on economic and social development, leading directly to less confidence in economic growth and a sharp drop in investment. The 1918 Spanish flu infected about 500 million people worldwide. The 1957 Asian influenza pandemic killed at least 1 million people. According to World Bank officials, the 1968 Hong Kong flu could cause global GDP to fall by $0.7 \%$ in the first year. The 2002 SARS caused a productivity loss of more than 40 billion US dollars. The epidemics hit trade and services hard. The World Bank estimates that the SARS epidemic caused 54 billion US dollars to the global economy, while the 2009 Influenza A (H1N1) pandemic caused between 45 and 55 billion US dollars in global losses. Besides the economic impact, the decline in human capital indirectly affected economic activity in the decades following the pandemic. In fact, the poor suffered the most significant impact, which exacerbated social inequality. Besides, the epidemic not only causes economic depression, but also causes patients and their families to be isolated and stigmatized, and suffering high psychological stress.

Researchers urgently need to establish mathematical models to predict pandemic trends and formulate better prevention, control, and rescue policies. Kermack and McKendrick (1927) build the first susceptible-infected-recovered (SIR) model to describe the spread of the epidemic. This SIR model has been widely extended to model different types of outbreaks. Li et al. (2020) add mobility data between cities into the SIR model to build a networked dynamic metapopulation model to infer critical epidemiological characteristics associated with COVID-19. Jia et al. (2020) use mobile-phone-data-based counts of about 11.5 million people egressing or transiting through the prefecture of Wuhan to build a risk source model to derive the geographic spread of COVID-19 statistically. Besides deterministic SIR models, stochastic SIR models are established based on the consideration transmission rate having stochastic perturbations. Bartlett (1956) formulates the first stochastic extensions of the SIR model using a stochastic jump process to describe the evolution of an epidemic. Iwata and Miyakoshi (2020) conduct simulations to estimate the impact of potential secondary outbreaks in a community outside China using a stochastic SEIR model. Various other stochastic extensions of the SIR models have been proposed (see, e.g. Brauer et al. 2019).

The continuous-time SIR (SEIR) or stochastic SIR (SEIR) models are often used to estimate the whole trend of the epidemic at its outbreaks. In order to deal with belief degrees, Liu (2007) builds uncertainty theory. Liu (2008) introduces a type of differential equation driven by Liu process concerned with the analysis of belief degree in a system. Yao and Chen (2013a) establish the numerical solutions for uncertain differential equations using the $\alpha$-path methods. Li et al. (2017) build an uncertain SIS models via uncertain differential equations. Fang et al. (2018) discuss $\alpha$-path of uncertain SIS epidemic model with standard incidence and demography. Li et al. (2018) compare the deterministic, stochastic, and uncertain SIS models. Furthermore, Li and Teng (2019) analyze an uncertain SIS epidemic model with nonlinear incidence and demography. Uncertain SIS model is a type of mathematical model to describe an epidemic like flu. For a new virus like COVID-19, SARS or H1N1, it lasts only for a short period and the recovered cases immune to the virus. Through this COVID-19 pandemic, we can see that the numbers of infected and recovered in the population are 
crucial to the development of the outbreak control. From the news, we know it is tough to attain accurate numbers of infected and recovered cases. The survey sponsored by Stanford University (Bendavid et al. 2020) shows the infection may be much more widespread than indicated by the number of confirmed cases. The study by Imperial College London (Unwin et al. 2020) finds hundreds of thousands more Massachusetts residents likely contracted COVID-19 than reported. Centers for Disease Control and Prevention (CDC) chief said COVID-19 cases may be 10 times higher than reported based on antibody tests on June 25, 2020.

This paper aims to build an uncertain SIR model via high-dimensional uncertain differential equations. As we know, high-dimensional uncertain differential equations are more flexible in applications. However, developing algorithms for solving highdimensional uncertain differential equations has been an exceedingly difficult task for a long time. Thus, we establish an $\alpha$-path-approached method for the proposed SIR model, estimate parameters using the method of moments, and give numerical methods to solve them. Finally, we employ the estimated parameters in the model to study the COVID-19 in Hubei province, China. The remainder of this paper is organized as follows. Preliminaries of uncertainty theory are recalled in Sect. 2. An uncertain SIR model based on high-dimensional uncertain differential equations is built in Sect. 3. Section 4 introduces $\alpha$-path and proves the theorem for numerical solution, and Sect. 5 estimates parameters and designs a 99-method to solve the proposed uncertain differential system. A calibration on uncertain SIR model is discussed in Sect. 6. A brief summary is included in Sect. 7.

\section{Preliminaries}

Uncertainty theory is a branch of mathematics based on normality, duality, subadditivity, and product axioms. It is founded by Liu (2007) to deal with belief degrees. The core concept in uncertainty theory is uncertain measure. The definition of uncertain measure is recalled as follows.

Definition 1 (Liu (2007)) Let $\mathcal{L}$ be a $\sigma$-algebra on a nonempty set $\Gamma$. A set function $\mathcal{M}: \mathcal{L} \rightarrow[0,1]$ is called an uncertain measure if it satisfies the following axioms:

Axiom 1: (Normality Axiom) $\mathcal{M}\{\Gamma\}=1$ for the universal set $\Gamma$.

Axiom 2: (Duality Axiom) $\mathcal{M}\{\Lambda\}+\mathcal{M}\left\{\Lambda^{c}\right\}=1$ for any event $\Lambda$.

Axiom 3: (Subadditivity Axiom) For every countable sequence of events $\Lambda_{1}, \Lambda_{2}, \ldots$, we have

$$
\mathcal{M}\left\{\bigcup_{i=1}^{\infty} \Lambda_{i}\right\} \leq \sum_{i=1}^{\infty} \mathcal{M}\left\{\Lambda_{i}\right\}
$$

Besides, the product uncertain measure on the product $\sigma$-algebra $\mathcal{L}$ is defined by Liu (2009) bellow. 
Axiom 4: (Product Axiom) Let $\left(\Gamma_{k}, \mathcal{L}_{k}, \mathcal{M}_{k}\right)$ be uncertainty spaces for $k=1,2, \ldots$ Then the product uncertain measure $\mathcal{M}$ is an uncertain measure satisfying

$$
\mathcal{M}\left\{\prod_{i=1}^{\infty} \Lambda_{k}\right\}=\bigwedge_{k=1}^{\infty} \mathcal{M}_{k}\left\{\Lambda_{k}\right\}
$$

where $\Lambda_{k}$ are arbitrarily chosen events from $\mathcal{L}_{k}$ for $k=1,2, \ldots$, respectively.

The triplet $(\Gamma, \mathcal{L}, \mathcal{M})$ is called an uncertainty space. Based on the axioms of uncertain measure, uncertainty theory is founded by Liu (2007) and refined by Liu (2010). An uncertain variable is a function from an uncertainty space $(\Gamma, \mathcal{L}, \mathcal{M})$ to the set of real numbers. The uncertainty distribution $\Phi$ of an uncertain variable $\xi$ is defined by

$$
\Phi(x)=\mathcal{M}\{\xi \leq x\}
$$

for any real number $x$. An uncertainty distribution $\Phi(x)$ is said to be regular if it is a continuous and strictly increasing function with respect to $x$ at which $0<\Phi(x)<1$, and

$$
\lim _{x \rightarrow-\infty} \Phi(x)=0, \lim _{x \rightarrow+\infty} \Phi(x)=1
$$

Definition 2 (Liu (2010)) Let $\xi$ be an uncertain variable with regular uncertainty distribution $\Phi(x)$. Then the inverse function $\Phi^{-1}(\alpha)$ is called the inverse uncertainty distribution of $\xi$.

Let $\xi$ be an uncertain variable with an uncertainty distribution $\Phi$. If the expected value exists, then it is proved by Liu (2010) that

$$
E[\xi]=\int_{0}^{1} \Phi^{-1}(\alpha) \mathrm{d} \alpha .
$$

Theorem 1 (Liu (2010)) Let $\xi$ be an uncertain variable with an uncertainty distribution $\Psi$. If $f$ is a strictly increasing function, then $\eta=f(\xi)$ is an uncertain variable with an inverse uncertainty distribution

$$
\Phi^{-1}(\alpha)=f\left(\Psi^{-1}(\alpha)\right)
$$

If $f$ is a strictly decreasing function, then $\eta=f(\xi)$ is an uncertain variable with an inverse uncertainty distribution

$$
\Phi^{-1}(\alpha)=f\left(\Psi^{-1}(1-\alpha)\right)
$$


An uncertain process is essentially a sequence of uncertain variables indexed by time. The study of uncertain process is started by Liu (2008).

Definition 3 (Liu (2008)) Let $(\Gamma, \mathcal{L}, \mathcal{M})$ be an uncertainty space and let $T$ be a totally ordered set (e.g. time). An uncertain process is a function $X_{t}(\gamma)$ from $T \times(\Gamma, \mathcal{L}, \mathcal{M})$ to the set of real numbers such that $\left\{X_{t} \in B\right\}$ is an event for any Borel set $B$ of real numbers at each time $t$.

Definition 4 (Liu (2009)) An uncertain process $C_{t}(t \geq 0)$ is said to be a Liu process if

(i) $C_{0}=0$ and almost all sample paths are Lipschitz continuous,

(ii) $C_{t}$ has stationary and independent increments,

(iii) every increment $C_{s+t}-C_{s}$ is a normal uncertain variable with expected value 0 and variance $t^{2}$, whose uncertainty distribution is

$$
\Phi(x)=\left(1+\exp \left(\frac{-\pi x}{\sqrt{3} t}\right)\right)^{-1}, x \in \Re .
$$

Based on Liu process, uncertain integral and uncertain differential are defined by Liu (2009), thus offering a theory of uncertain calculus. An uncertain differential equation driven by Liu process is defined as follows.

Definition 5 (Liu (2008)) Suppose $C_{t}$ is a Liu process, and $f$ and $g$ are some given functions. Then

$$
\mathrm{d} X_{t}=f\left(t, X_{t}\right) \mathrm{d} t+g\left(t, X_{t}\right) \mathrm{d} C_{t}
$$

is called an uncertain differential equation. A solution is an uncertain process $X_{t}$ that satisfies (1) identically in $t$.

The existence and uniqueness theorem for uncertain differential equations is proved by Chen and Liu (2010). Uncertain differential equation theory has been applied in the fields such as finance, population growth model, dynamic game theory, and optimal control (Liu 2015). The concept of $\alpha$-path is introduced by Yao and Chen (2013a). The solution to an uncertain differential equation is equivalent to a group of $\alpha$-path solutions to related ordinary differential equations. Besides, Chen and Gao (2018) further studied the $\alpha$-path for nested differential equations. The definition of $\alpha$-path is as follows.

Definition 6 (Yao and Chen (2013a)) The $\alpha$-path $(0<\alpha<1)$ of an uncertain differential equation

$$
\mathrm{d} X_{t}=f\left(t, X_{t}\right) \mathrm{d} t+g\left(t, X_{t}\right) \mathrm{d} C_{t}
$$

is a deterministic function $X_{t}^{\alpha}$ with respect to $t$ that solves the corresponding ordinary differential equation

$$
\mathrm{d} X_{t}^{\alpha}=f\left(t, X_{t}^{\alpha}\right) \mathrm{d} t+\left|g\left(t, X_{t}^{\alpha}\right)\right| \Phi^{-1}(\alpha) \mathrm{d} t
$$


where $\Phi^{-1}(\alpha)$ is the inverse uncertainty distribution of standard normal uncertain variable, i.e.,

$$
\Phi^{-1}(\alpha)=\frac{\sqrt{3}}{\pi} \ln \frac{\alpha}{1-\alpha}, \quad 0<\alpha<1
$$

The following theorem shows that the solution of an uncertain differential equation is related to a class of ordinary differential equations.

Theorem 2 (Yao and Chen (2013a)) Let $X_{t}$ and $X_{t}^{\alpha}$ be the solution and $\alpha$-path of the uncertain differential equation

$$
\mathrm{d} X_{t}=f\left(t, X_{t}\right) \mathrm{d} t+g\left(t, X_{t}\right) \mathrm{d} C_{t},
$$

respectively. Then

$$
\begin{aligned}
& \mathcal{M}\left\{X_{t} \leq X_{t}^{\alpha}, \forall t>0\right\}=\alpha, \\
& \mathcal{M}\left\{\left\{X_{t}>X_{t}^{\alpha}, \forall t>0\right\}=1-\alpha .\right.
\end{aligned}
$$

\section{Uncertain SIR model}

During a pandemic, people who recover will be likely immune to it. The infected and recovered numbers of cases are essential variables that determine the pandemic trend. The fact that many cases are initially asymptomatic makes it difficult to ascertain the exact number of individuals infected with COVID-19. Surveys indicate that the confirmed infected cased number denoted by $I_{t}$ in the whole population is a significant difference from the actual infected number. Tests found that a considerable amount of people have already obtained COVID-19 antibodies without any symptoms. The real number for $R_{t}$ is more difficult to estimate because of these asymptomatic infections. In order to indeterminism in the pandemic, the uncertain differential equation is employed to build an uncertain SIR model. In our uncertain SIR model, originated by Kermack and McKendrick (1927), the population $N_{t}$ is divided into three categories susceptible $S_{t}$, infected $I_{t}$, and recovered $R_{t}$. We use Liu processes to model diffusion sources that capture variability in exposures and potential mismeasurement of the numbers of infected and recovered. The uncertain SIR pandemic model is introduced as follows,

$$
\begin{aligned}
\mathrm{d} S_{t} & =\left(a-\beta I_{t} S_{t}-\mu S_{t}\right) \mathrm{d} t+\sigma_{S} S_{t} \mathrm{~d} C_{t}^{S}, \\
\mathrm{~d} I_{t} & =\left(\beta I_{t} S_{t}-(\lambda+\epsilon+\mu) I_{t}\right) \mathrm{d} t+\sigma_{I} I_{t} \mathrm{~d} C_{t}^{I}, \\
\mathrm{~d} R_{t} & =\left(\lambda I_{t}-\mu R_{t}\right) \mathrm{d} t+\sigma_{R} R_{t} \mathrm{~d} C_{t}^{R}
\end{aligned}
$$

where $a$ is the influx of individuals into the susceptible; $\beta$ is the disease transmission coefficient; $\mu$ represents the natural mortality rate; $\epsilon$ represents additional death rate related to pandemic infection; $\lambda$ represents the rate of recovery from infection; $C_{t}^{S}$, $C_{t}^{I}$, and $C_{t}^{R}$ are independent Liu processes; $\sigma_{S}, \sigma_{I}$, and $\sigma_{R}$ are positive numbers which represent the volatility of the diffusion processes, respectively. 
The SIR model studies the transmission dynamics of the disease and the resulting population flows among the compartments. Since the number of the total population is deterministic, we only put two diffusion sources into modeling $I_{t}$ and $R_{t}$, and let the rest of the population be susceptible. Namely, we set $\sigma_{S}=0$ in the following parts. In our model, we just introduce $I_{t}$ as the infection cases. Actually, more sub-groups can be added into the system like hospitalizations cases, ICU cases, and ventilator cases in SIR models, as discussed by Hill et al. (2020). There is no increase in the technical difficulty if we add more items into our model. The theorems proved in the following sections also hold under these extensions. Different from other uncertain models where the transmission rate or recovery rate are modeled via Liu processes, multiple diffusion sources are introduced in our model to characterize various sources of in-deterministic.

Next, we compare our model with existing uncertain epidemic models. First, our model is different from the uncertain SIS epidemic model proposed by Li et al. (2017). The uncertain SIS epidemic model does not involve the recovered cases, which is immune to the virus in our model. Without recovered cases, the proposed uncertain SIS model could be used to describe common seasonal influenza. Pandemic is different from this seasonal influenza, which lasts for a short period, mostly less than two years. The following studies by Fang et al. (2018), Li et al. (2018), and Li and Teng (2019) all focus on uncertain SIS models other than the SIR model. Secondly, our model is different from the uncertain SEIAR model introduced by Jia and Chen (2020). It is undeniable that the deterministic SEIR model could indeed degenerate into an SIR model. The diffusion sources in their uncertain SEIR model have specific correlations. Even if some volatility parameters in the uncertain SEIAR model become zero, our model is still not a special case of their model. There is no $\alpha$-path solution for this uncertain SEIAR model, which prevents its further applications in high-dimensional situations. In the next section, we derive the $\alpha$-path solutions of our model and estimate parameters for the proposed model with application to COVID-19 pandemic.

\section{Uncertain $\alpha$-paths for SIR pandemic model}

It is obvious that the proposed uncertain SIR pandemic model has no analytic solutions. In order to solve this uncertain differential system, we plan to promote the uncertain $\alpha$ path method to develop numerical solutions. Now, we will discuss the $\alpha$-paths for the uncertain SIR pandemic model. For the uncertain system (4), the uncertain $\alpha$-path is defined based on each uncertain differential equation. In fact, if the parameter $\sigma_{S} \neq 0$, the system (4) does not have $\alpha$-path. Now, we will focus on the condition that $\sigma_{S}=0$ and prove the $\alpha$-path for it.

Definition 7 The $\alpha$-path $(0<\alpha<1)$ of the uncertain differential system (4) with initial values $S(0), I(0)$ and $R(0)$ are deterministic functions $S_{t}^{\alpha}, I_{t}^{\alpha}$ and $R_{t}^{\alpha}$ with respect to $t$, respectively, that solve the corresponding ordinary differential equations

$$
\begin{aligned}
\mathrm{d} S_{t}^{1-\alpha} & =\left(a-\beta I_{t}^{\alpha} S_{t}^{1-\alpha}-\mu S_{t}^{1-\alpha}\right) \mathrm{d} t \\
\mathrm{~d} I_{t}^{\alpha} & =\left(\beta I_{t}^{\alpha} S_{t}^{1-\alpha}-(\lambda+\epsilon+\mu) I_{t}^{\alpha}+\sigma_{I} I_{t}^{\alpha} \Phi^{-1}(\alpha)\right) \mathrm{d} t,
\end{aligned}
$$




$$
\mathrm{d} R_{t}^{\alpha}=\left(\lambda I_{t}^{\alpha}-\mu R_{t}^{\alpha}+\sigma_{R} R_{t}^{\alpha} \Phi^{-1}(\alpha)\right) \mathrm{d} t
$$

where $\Phi^{-1}(\alpha)$ is the inverse uncertainty distribution of standard normal uncertain variable, i.e.,

$$
\Phi^{-1}(\alpha)=\frac{\sqrt{3}}{\pi} \ln \frac{\alpha}{1-\alpha}, \quad 0<\alpha<1
$$

In order to study the $\alpha$-path of the proposed uncertain differential equations, a useful lemma is first introduced here. For a system with several differential equations, there is no general comparison theorem. We need to apply the comparison theorem to each differential equation.

Lemma 1 Assume that $f(t, x, y)$ and $g(t, x)$ are continuous functions. Let $\phi_{t}$ be a solution of the ordinary differential equation

$$
\frac{\mathrm{d} x}{\mathrm{~d} t}=f(t, x, \boldsymbol{y})+K|g(t, x)|, \quad x(0)=x_{0}
$$

where $K$ is a real number. Let $\psi_{t}$ be a solution of the ordinary differential equation

$$
\frac{\mathrm{d} x}{\mathrm{~d} t}=f(t, x, y)+k_{t} g(t, x), \quad x(0)=x_{0}
$$

where $k_{t}$ is a real function satisfying

(i) If $k_{t} g(t, x) \leq K|g(t, x)|$ for $t \in[0, T]$, then $\psi_{t} \leq \phi_{t}$,

(ii) If $k_{t} g(t, x)>K|g(t, x)|$ for $t \in[0, T]$, then $\psi_{t}>\phi_{t}$.

Theorem 3 Let $S_{t}, I_{t}, R_{t}$ and $S_{t}^{\alpha}, I_{t}^{\alpha}, R_{t}^{\alpha}$ be the solutions and $\alpha$-paths of the uncertain differential Eqs. $(4 \mathrm{a}-4 \mathrm{c})$ when $\sigma_{S}=0$, respectively. Then

$$
\begin{aligned}
& \mathcal{M}\left\{S_{t} \geq S_{t}^{1-\alpha}, I_{t} \leq I_{t}^{\alpha}, R_{t} \leq R_{t}^{\alpha}, \forall t>0\right\}=\alpha, \\
& \mathcal{M}\left\{S_{t}<S_{t}^{1-\alpha}, I_{t}>I_{t}^{\alpha}, R_{t}>R_{t}^{\alpha}, \forall t>0\right\}=1-\alpha .
\end{aligned}
$$

Proof Note that $\sigma_{I} I_{t}^{\alpha} \geq 0$ and $\sigma_{R} R_{t}^{\alpha} \geq 0, \forall \alpha \in[0,1]$. Write

$$
\Lambda_{1}=\left\{\gamma \mid \frac{\mathrm{d} C_{t}^{I}(\gamma)}{\mathrm{d} t} \leq \Phi^{-1}(\alpha), \frac{\mathrm{d} C_{t}^{R}(\gamma)}{\mathrm{d} t} \leq \Phi^{-1}(\alpha), \forall t \in(0, u]\right\}
$$

where $\Phi^{-1}$ is the inverse uncertainty distribution of $\mathcal{N}(0,1)$ for any given $u>0$. Since both $C_{t}^{I}$ and $C_{t}^{R}$ are independent increment processes. We get $\mathcal{M}\left\{\left\{\Lambda_{1}\right\}=\alpha\right.$. For any $\gamma \in \Lambda_{1}$, we have

$$
\left.a-\left(\beta I_{t}(\gamma) S_{t}(\gamma)+\mu S_{t}(\gamma)\right) \geq a-\left(\beta I_{t}^{\alpha} S_{t}^{1-\alpha}+\mu S_{t}^{1-\alpha}\right)\right),
$$




$$
\begin{gathered}
\beta I_{t}(\gamma) S_{t}(\gamma)-(\lambda+\epsilon+\mu) I_{t}(\gamma)+\sigma_{S} I_{t}(\gamma) \frac{\mathrm{d} C_{t}^{I}(\gamma)}{\mathrm{d} t} \leq \beta I_{t}^{\alpha} S_{t}^{1-\alpha} \\
-(\lambda+\epsilon+\mu) I_{t}^{\alpha}+\sigma_{S} I_{t}^{\alpha} \Phi^{-1}(\alpha), \\
\lambda I_{t}(\gamma)-\mu R_{t}(\gamma)+\sigma_{R} R_{t}(\gamma) \frac{\mathrm{d} C_{t}^{R}(\gamma)}{\mathrm{d} t} \leq \lambda I_{t}^{\alpha}-\mu R_{t}^{\alpha}+\sigma_{R} R_{t}^{\alpha} \Phi^{-1}(\alpha) .
\end{gathered}
$$

It follows from Lemma 1 that $S_{t}(\gamma) \geq S_{t}^{1-\alpha}, I_{t}(\gamma) \leq I_{t}^{\alpha}$ and $R_{t}(\gamma) \leq R_{t}^{\alpha}$. Thus, we get

$$
\Lambda_{1} \subset\left\{S_{t} \geq S_{t}^{1-\alpha}, I_{t} \leq I_{t}^{\alpha}, R_{t} \leq R_{t}^{\alpha}, \forall t>0\right\}
$$

It follows from the independence of $C_{t}^{I}$ and $C_{t}^{R}$ that

$$
\mathcal{M}\left\{S_{t} \geq S_{t}^{1-\alpha}, I_{t} \leq I_{t}^{\alpha}, R_{t} \leq R_{t}^{\alpha}, \forall t>0\right\} \geq \mathcal{M}\left\{\Lambda_{1}\right\}=\alpha .
$$

On the other hand, write

$$
\Lambda_{2}=\left\{\gamma \mid \frac{\mathrm{d} C_{t}^{I}(\gamma)}{\mathrm{d} t}>\Phi^{-1}(\alpha), \frac{\mathrm{d} C_{t}^{R}(\gamma)}{\mathrm{d} t}>\Phi^{-1}(\alpha) \text { for } t \in(0, u]\right\} .
$$

Then, with the help of independence of $C_{t}^{I}$ and $C_{t}^{R}$, we get $\mathcal{M}\left\{\Lambda_{2}\right\}=1-\alpha$. For any $\gamma \in \Lambda_{2}$, we have

$$
\begin{gathered}
\left.a-\left(\beta I_{t}(\gamma) S_{t}(\gamma)+\mu S_{t}(\gamma)\right)<a-\left(\beta I_{t}^{\alpha} S_{t}^{1-\alpha}+\mu S_{t}^{1-\alpha}\right)\right), \\
\beta I_{t}(\gamma) S_{t}(\gamma)-(\lambda+\epsilon+\mu) I_{t}(\gamma)+\sigma_{S} I_{t}(\gamma) \frac{\mathrm{d} C_{t}^{I}(\gamma)}{\mathrm{d} t}>\beta I_{t}^{\alpha} S_{t}^{1-\alpha} \\
-(\lambda+\epsilon+\mu) I_{t}^{\alpha}+\sigma_{S} I_{t}^{\alpha} \Phi^{-1}(\alpha), \\
\lambda I_{t}(\gamma)-\mu R_{t}(\gamma)+\sigma_{R} R_{t}(\gamma) \frac{\mathrm{d} C_{t}^{R}(\gamma)}{\mathrm{d} t}>\lambda I_{t}^{\alpha}-\mu R_{t}^{\alpha}+\sigma_{R} R_{t}^{\alpha} \Phi^{-1}(\alpha) .
\end{gathered}
$$

It follows from Lemma 1 that $S_{t}(\gamma)<S_{t}^{1-\alpha}, I_{t}(\gamma)>I_{t}^{\alpha}$ and $R_{t}(\gamma)>R_{t}^{\alpha}$. Thus, we get

$$
\Lambda_{2} \subset\left\{S_{t}<S_{t}^{1-\alpha}, I_{t}>I_{t}^{\alpha}, R_{t}>R_{t}^{\alpha}, \forall t>0\right\} .
$$

It follows from the independence of $C_{t}^{I}$ and $C_{t}^{R}$ that

$$
\mathcal{M}\left\{S_{t}<S_{t}^{1-\alpha}, I_{t}>I_{t}^{1-\alpha}, R_{t}>R_{t}^{1-\alpha}, \forall t>0\right\} \geq \mathcal{M}\left\{\Lambda_{2}\right\}=1-\alpha .
$$

By Eqs. (8) and (9), we have

$$
\mathcal{M}\left\{S_{t} \geq S_{t}^{1-\alpha}, I_{t} \leq I_{t}^{\alpha}, R_{t} \leq R_{t}^{\alpha}, \forall t>0\right\}=\alpha
$$

The theorem is verified. 
Theorem 4 Let $S_{t}, I_{t}, R_{t}$ and $S_{t}^{\alpha}, I_{t}^{\alpha}, R_{t}^{\alpha}$ be the solutions and $\alpha$-paths of the uncertain differential Eqs. $(4 \mathrm{a}-4 \mathrm{c})$ when $\sigma_{S}=0$, respectively. Then we have

$$
\begin{aligned}
\mathcal{M}\left\{S_{t} \leq S_{t}^{\alpha}, \forall t>0\right\} & =\alpha, \mathcal{M}\left\{S_{t}>S_{t}^{\alpha}, \forall t>0\right\}=1-\alpha, \\
\mathcal{M}\left\{I_{t} \leq I_{t}^{\alpha}, \forall t>0\right\} & =\alpha, \mathcal{M}\left\{I_{t}>I_{t}^{\alpha}, \forall t>0\right\}=1-\alpha, \\
\mathcal{M}\left\{R_{t} \leq R_{t}^{\alpha}, \forall t>0\right\} & =\alpha, \mathcal{M}\left\{R_{t}>R_{t}^{\alpha}, \forall t>0\right\}=1-\alpha .
\end{aligned}
$$

Proof Note that

$$
\left\{S_{t} \geq S_{t}^{1-\alpha}, I_{t} \leq I_{t}^{\alpha}, R_{t} \leq R_{t}^{\alpha}, \forall t>0\right\} \subset\left\{S_{t} \geq S_{t}^{1-\alpha}, \forall t>0\right\}
$$

and

$$
\left\{S_{t}<S_{t}^{1-\alpha}, I_{t}>I_{t}^{\alpha}, R_{t}>R_{t}^{\alpha}, \forall t>0\right\} \subset\left\{S_{t}<S_{t}^{1-\alpha}, \forall t>0\right\} .
$$

It follows from Eqs. (8) and (9) that

$$
\mathcal{M}\left\{S_{t} \geq S_{t}^{1-\alpha}, \forall t>0\right\} \geq \mathcal{M}\left\{S_{t} \geq S_{t}^{1-\alpha}, I_{t} \leq I_{t}^{\alpha}, R_{t} \leq R_{t}^{\alpha}, \forall t>0\right\}=\alpha
$$

and

$$
\mathcal{M}\left\{S_{t}<S_{t}^{1-\alpha}, \forall t>0\right\} \geq \mathcal{M}\left\{S_{t}<S_{t}^{1-\alpha}, I_{t}>I_{t}^{\alpha}, R_{t}>R_{t}^{\alpha}, \forall t>0\right\}=1-\alpha
$$

The union of this two disjoint sets $\left\{S_{t} \geq S_{t}^{1-\alpha}, \forall t>0\right\}$ and $\left\{S_{t}<S_{t}^{1-\alpha}, \forall t>0\right\}$ is subset of the universal set $\Gamma$. Since

$$
\mathcal{M}\left\{S_{t} \geq S_{t}^{1-\alpha}, \forall t>0\right\}+\mathcal{M}\left\{S_{t}<S_{t}^{1-\alpha}, \forall t>0\right\} \leq 1,
$$

we have

$$
\mathcal{M}\left\{S_{t} \geq S_{t}^{1-\alpha}, \forall t>0\right\}=\alpha, \mathcal{M}\left\{S_{t}<S_{t}^{1-\alpha}, \forall t>0\right\}=1-\alpha .
$$

The proof for $I_{t}$ and $R_{t}$ is analogous. The theorem is proved.

Theorem 5 Let $S_{t}, I_{t}, R_{t}$ and $S_{t}^{\alpha}, I_{t}^{\alpha}, R_{t}^{\alpha}$ be the solutions and $\alpha$-paths of the uncertain differential Eqs. (4a-4c) when $\sigma_{S}=0$, respectively. Then $I_{t}$ and $R_{t}$ have inverse uncertainty distributions

$$
\Phi_{t}^{-1}(\alpha)=I_{t}^{\alpha} \text { and } \Psi_{t}^{-1}(\alpha)=R_{t}^{\alpha}, \forall \alpha \in(0,1)
$$

respectively. And we have the expected values

$$
E\left[I_{t}\right]=\int_{0}^{1} I_{t}^{\alpha} \mathrm{d} \alpha \text { and } E\left[R_{t}\right]=\int_{0}^{1} R_{t}^{\alpha} \mathrm{d} \alpha .
$$


Proof For any given time $T$, it follows from Theorem 4 that

$$
\begin{aligned}
& \left\{I_{t} \leq I_{t}^{\alpha}\right\} \supset\left\{I_{t} \leq I_{t}^{\alpha}, \forall t>0\right\}, \\
& \left\{I_{t}>I_{t}^{\alpha}\right\} \supset\left\{I_{t}>I_{t}^{\alpha}, \forall t>0\right\} .
\end{aligned}
$$

We have

$$
\begin{aligned}
& \mathcal{M}\left\{I_{t} \leq I_{t}^{\alpha}\right\} \geq\left\{I_{t} \leq I_{t}^{\alpha}, \quad \forall t>0\right\}=\alpha, \\
& \mathcal{M}\left\{I_{t}>I_{t}^{\alpha}\right\} \geq\left\{I_{t}>I_{t}^{\alpha}, \quad \forall t>0\right\}=1-\alpha .
\end{aligned}
$$

It follows from the duality of uncertain measure that

$$
\mathcal{M}\left\{I_{t} \leq I_{t}^{\alpha}\right\}+\mathcal{M}\left\{I_{t}>I_{t}^{\alpha}\right\}=1 .
$$

Thus, we have

$$
\begin{aligned}
& \mathcal{M}\left\{I_{t} \leq I_{t}^{\alpha}\right\}=\alpha, \\
& \mathcal{M}\left\{I_{t}>I_{t}^{\alpha}\right\}=1-\alpha .
\end{aligned}
$$

Furthermore, we obtain

$$
E\left[I_{t}\right]=\int_{0}^{1} I_{t}^{\alpha} \mathrm{d} \alpha .
$$

Proving for $R_{t}$ is completely analogous.

Theorem 6 Let $S_{t}, I_{t}, R_{t}$ and $S_{t}^{\alpha}, I_{t}^{\alpha}, R_{t}^{\alpha}$ be the solutions and $\alpha$-paths of the uncertain differential Eqs. (4a-4c) when $\sigma_{S}=0$, respectively. Then the supremum process

$$
Y_{t}=\max _{0 \leq t \leq T} I_{t}
$$

has $\alpha$-paths

$$
Y_{t}^{\alpha}=\max _{0 \leq t \leq T} I_{t}^{\alpha}
$$

Proof For a sample path $I_{t}(\gamma)$ such that $I_{T}(\gamma) \leq I_{t}^{\alpha}$ for any time $T$, we have

$$
\max _{0 \leq t \leq T} I_{t}(\gamma) \leq \max _{0 \leq t \leq T} I_{t}^{\alpha}
$$


It implies

$$
\left\{Y_{T} \leq \max _{0 \leq t \leq T} I_{t}^{\alpha}, \forall T>0\right\} \supset\left\{I_{T} \leq I_{T}^{\alpha}, \forall T>0\right\}
$$

Using the monotonicity theorem of uncertain measure, we have

$$
\mathcal{M}\left\{Y_{T} \leq \max _{0 \leq t \leq T} I_{t}^{\alpha}, \forall T>0\right\} \geq \mathcal{M}\left\{I_{T} \leq I_{T}^{\alpha}, \forall T>0\right\}=\alpha .
$$

Similarly, we have

$$
\mathcal{M}\left\{Y_{T}>\max _{0 \leq t \leq T} I_{t}^{\alpha}, \forall T>0\right\} \geq \mathcal{M}\left\{I_{T}>I_{T}^{\alpha}, \forall T>0\right\}=1-\alpha .
$$

It follows from the duality of uncertain measure that

$$
\mathcal{M}\left\{Y_{T}>\max _{0 \leq t \leq T} I_{t}^{\alpha}, \forall T>0\right\}+\mathcal{M}\left\{Y_{T} \leq \max _{0 \leq t \leq T} I_{t}^{\alpha}, \forall T>0\right\}=1
$$

Thus, we have

$$
\begin{aligned}
& \mathcal{M}\left\{Y_{T} \leq \max _{0 \leq t \leq T} I_{t}^{\alpha}, \forall T>0\right\}=\alpha, \\
& \mathcal{M}\left\{Y_{T}>\max _{0 \leq t \leq T} I_{t}^{\alpha}, \forall T>0\right\}=1-\alpha .
\end{aligned}
$$

So $Y_{T}$ has $\alpha$-paths

$$
Y_{T}^{\alpha}=\max _{0 \leq t \leq T} I_{t}^{\alpha}, \alpha \in(0,1)
$$

Theorem 7 Let $S_{t}, I_{t}, R_{t}$ and $S_{t}^{\alpha}, I_{t}^{\alpha}, R_{t}^{\alpha}$ be the solutions and $\alpha$-paths of the uncertain differential Eqs. $(4 \mathrm{a}-4 \mathrm{c})$ when $\sigma_{S}=0$, respectively. Then the time integral process

$$
Z_{T}=\int_{0}^{T} R_{t} \mathrm{~d} t
$$

has $\alpha$-paths

$$
Z_{T}^{\alpha}=\int_{0}^{T} R_{t}^{\alpha} \mathrm{d} t
$$

Proof For a sample path $R_{T}(\gamma)$ such that $R_{T}(\gamma) \leq R_{T}^{\alpha}$ for any time $T$, we have 


$$
\int_{0}^{T} R_{t}(\gamma) \mathrm{d} t \leq \int_{0}^{T} R_{t}^{\alpha} \mathrm{d} t
$$

It implies

$$
\left\{\int_{0}^{T} R_{t} \mathrm{~d} t \leq \int_{0}^{T} R_{t}^{\alpha} \mathrm{d} t, \forall T>0\right\} \supset\left\{R_{T} \leq R_{T}^{\alpha}, \forall T>0\right\}
$$

Using the monotonicity theorem of uncertain measure, we have

$$
\mathcal{M}\left\{\int_{0}^{T} R_{t} \mathrm{~d} t \leq \int_{0}^{T} R_{t}^{\alpha} \mathrm{d} t, \forall T>0\right\} \geq \mathcal{M}\left\{R_{T} \leq R_{T}^{\alpha}, \forall T>0\right\}=\alpha .
$$

In the same way, we obtain

$$
\mathcal{M}\left\{\int_{0}^{T} R_{t} \mathrm{~d} t>\int_{0}^{T} R_{t}^{\alpha} \mathrm{d} t, \forall T>0\right\} \geq \mathcal{M}\left\{R_{T}>R_{T}^{\alpha}, \forall T>0\right\}=1-\alpha .
$$

It follows from duality axiom of uncertain measure that

$$
\begin{aligned}
& \mathcal{M}\left\{\int_{0}^{T} R_{t} \mathrm{~d} t \leq \int_{0}^{T} R_{t}^{\alpha} \mathrm{d} t, \forall T>0\right\}=\alpha, \\
& \mathcal{M}\left\{\int_{0}^{T} R_{t} \mathrm{~d} t>\int_{0}^{T} R_{t}^{\alpha} \mathrm{d} t, \forall T>0\right\}=1-\alpha .
\end{aligned}
$$

Thus, we have the $\alpha$-paths of $Z_{T}$

$$
Z_{T}^{\alpha}=\int_{0}^{T} R_{t}^{\alpha} \mathrm{d} t
$$

Theorem 8 Let $S_{t}, I_{t}, R_{t}$ and $S_{t}^{\alpha}, I_{t}^{\alpha}, R_{t}^{\alpha}$ be the solutions and $\alpha$-paths of the uncertain differential Eqs. (4a-4c) when $\sigma_{S}=0$, respectively. Then for the monotone function $J$, we have

$$
\begin{aligned}
E\left[J\left(I_{t}\right)\right] & =\int_{0}^{1} J\left(I_{t}^{\alpha}\right) \mathrm{d} \alpha, \\
E\left[J\left(R_{t}\right)\right] & =\int_{0}^{1} J\left(R_{t}^{\alpha}\right) \mathrm{d} \alpha .
\end{aligned}
$$

Proof At first, it follows from Theorem 4 that $I_{t}$ has an uncertainty distributions $\Psi_{t}^{-1}(\alpha)=I_{t}^{\alpha}$. When $J$ is a strictly increasing function, it follows from Theorem 1 that $J\left(I_{t}\right)$ has an inverse uncertainty distribution $\Phi_{t}^{-1}(\alpha)=J\left(I_{t}^{\alpha}\right)$. Thus, we have 


$$
E\left[J\left(I_{t}\right]=\int_{0}^{1} \Phi_{t}^{-1}(\alpha) \mathrm{d} \alpha=\int_{0}^{1} J\left(I_{t}^{\alpha}\right) \mathrm{d} \alpha\right.
$$

When $J$ is a strictly decreasing function, it follows from Theorem 1 that $J\left(I_{t}\right)$ has an inverse uncertainty distribution $\Phi_{t}^{-1}(\alpha)=J\left(I_{t}^{1-\alpha}\right)$. Thus, we have

$$
E\left[J\left(I_{t}\right]=\int_{0}^{1} \Phi_{t}^{-1}(1-\alpha) \mathrm{d} \alpha=\int_{0}^{1} J\left(I_{t}^{1-\alpha}\right) \mathrm{d} \alpha=\int_{0}^{1} J\left(I_{t}^{\alpha}\right) \mathrm{d} \alpha .\right.
$$

The proof for Eq. (13b) is completely analogous. The theorem is thus proved.

In the pandemic, it is useful to use

$$
E\left[\max _{0 \leq t \leq T} I_{t}\right]=\int_{0}^{1} \max _{0 \leq t \leq T} I_{t}^{\alpha} \mathrm{d} \alpha
$$

to estimate the demand of medical equipment like personal protective equipment (PPE), ICU beds and ventilators. The value

$$
E\left[\int_{0}^{T} R_{t} \mathrm{~d} t\right]=\int_{0}^{1} \int_{0}^{T} R_{t}^{\alpha} \mathrm{d} t \mathrm{~d} \alpha
$$

is also a useful number to help formulate reopen policies. In fact, the solutions to uncertain differential Eqs. $(4 \mathrm{a}-4 \mathrm{c})$ are uncertain contour processes. The above results could also be proved via the principles provided by Yao (2015).

\section{Numerical method}

In this section, we will first estimate parameters for the uncertain SIR model and then introduce numerical methods to solve $\alpha$-path solutions.

\subsection{Parameter estimation}

We will employ the method proposed by Yao and Liu (2020) to estimate the parameters in our uncertain SIR model. The basic idea is to set the empirical moments of the functions of the parameters and the observed data equal to the moments of the standard normal uncertainty distribution. Let $i=1,2 \ldots, n$ be the days after the public data of infected and recovered numbers available. Compare to the whole population, the infected number is very low. For model simplification, we set $S(i)=N$ where $N$ is the whole population number. The uncertain SIR system has the following discrete form

$$
\begin{aligned}
& I(i+1)=I(i)+\beta I(i) N-(\lambda+\epsilon+\mu) I(i)+\sigma_{I} I(i)\left(C^{I}(i+1)-C^{I}(i)(14 \mathrm{a})\right. \\
& R(i+1)=R(i)+\lambda I(i)-\mu R(i)+\sigma_{R} R(i)\left(C^{R}(i+1)-C^{R}(i)\right) .
\end{aligned}
$$


In the above difference equations, the parameters $a, \epsilon$, and $\mu$ could be obtained from public data resources. We only need to estimate the parameters $\beta, \lambda, \sigma_{I}$, and $\sigma_{R}$. With the help of Yao and Liu (2020), we build the following two statistics

$$
\begin{aligned}
H_{i}^{I} & =\frac{(I(i+1)-I(i))-(\beta I(i) N-(\lambda+\epsilon+\mu) I(i))}{\sigma_{I} I(i)} \sim \mathcal{N}(0,1), \\
H_{i}^{R} & =\frac{R(i+1)-R(i)-(\lambda I(i)-\mu R(i))}{\sigma_{R} R(i)} \sim \mathcal{N}(0,1) .
\end{aligned}
$$

For the estimating of parameters $\beta, \lambda, \sigma_{I}$, and $\sigma_{R}$, the Eqs. (15a-15b) can be regarded as $n-1$ samples of a standard normal uncertainty distribution $\mathcal{N}(0,1)$. The $k$-th sample moments equal the $k$-th population moments

$$
\begin{aligned}
& \frac{1}{n-1} \sum_{i=1}^{n-1}\left(H_{i}^{I}\right)^{k}=\left(\frac{\sqrt{3}}{\pi}\right)^{k} \int_{0}^{1}\left(\ln \frac{\alpha}{1-\alpha}\right)^{k} \mathrm{~d} \alpha, \\
& \frac{1}{n-1} \sum_{i=1}^{n-1}\left(H_{i}^{R}\right)^{k}=\left(\frac{\sqrt{3}}{\pi}\right)^{k} \int_{0}^{1}\left(\ln \frac{\alpha}{1-\alpha}\right)^{k} \mathrm{~d} \alpha .
\end{aligned}
$$

We use the 1 st and 2nd moments to estimate the four parameters with

$$
\begin{aligned}
& \frac{1}{n-1} \sum_{i=1}^{n-1} H_{i}^{I}=0, \frac{1}{n-1} \sum_{i=1}^{n-1}\left(H_{i}^{I}\right)^{2}=1, \\
& \frac{1}{n-1} \sum_{i=1}^{n-1} H_{i}^{R}=0, \frac{1}{n-1} \sum_{i=1}^{n-1}\left(H_{i}^{R}\right)^{2}=1 .
\end{aligned}
$$

Solving the above equations, we get

$$
\begin{aligned}
\hat{\lambda} & =\left(\frac{1}{n-1} \sum_{i=1}^{n-1} \frac{R(i+1)-R(i)+\mu R(i)}{R(i)}\right) /\left(\frac{1}{n-1} \sum_{i=1}^{n-1} \frac{I(i)}{R(i)}\right) \\
\hat{\sigma}_{R} & =\sqrt{\frac{1}{n-1} \sum_{i=1}^{n-1}\left(\frac{R(i+1)-R(i)-(\hat{\lambda} I(i)-\mu R(i))}{R(i)}\right)^{2}} \\
\hat{\beta} & =\frac{1}{n-1} \sum_{i=1}^{n-1} \frac{(I(i+1)-I(i))+(\hat{\lambda}+\epsilon+\mu) I(i))}{I(i) N}, \\
\hat{\sigma}_{I} & =\sqrt{\frac{1}{n-1} \sum_{i=1}^{n-1}\left(\frac{(I(i+1)-I(i))-(\hat{\beta} I(i) N-(\hat{\lambda}+\epsilon+\mu) I(i))}{I(i)}\right)^{2}}
\end{aligned}
$$




\subsection{Numerical methods for $\alpha$-path}

Based on the previous theorems, a 99-method for solving a nested uncertain differential equation is designed as below.

Step 0: Fix a time $T$ and set $\alpha=0$.

Step 1: Set $\alpha \leftarrow \alpha+0.01$.

Step 2: Solve the corresponding ordinary differential equations

$$
\begin{aligned}
\mathrm{d} S_{t}^{1-\alpha} & =\left(a-\beta I_{t}^{\alpha} S_{t}^{1-\alpha}-\mu S_{t}^{1-\alpha}\right) \mathrm{d} t \\
\mathrm{~d} I_{t}^{\alpha} & =\left(\left(\beta I_{t}^{\alpha} S_{t}^{1-\alpha}-(\lambda+\epsilon+\mu) I_{t}^{\alpha}\right)+\sigma_{I} \Phi^{-1}(\alpha) I_{t}^{\alpha}\right) \mathrm{d} t, \\
\mathrm{~d} R_{t}^{\alpha} & =\left(\left(\lambda I_{t}^{\alpha}-\mu R_{t}^{\alpha}\right)+\sigma_{R} \Phi^{-1}(\alpha) R_{t}^{\alpha}\right) \mathrm{d} t, \\
\Phi^{-1}(\alpha) & =\frac{\sqrt{3}}{\pi} \ln \frac{\alpha}{1-\alpha}, t \in(0, T] .
\end{aligned}
$$

Then we obtain $S_{t}^{\alpha}, I_{t}^{\alpha}$, and $R_{t}^{\alpha}$ with initial conditions $S(0)=S_{0}, I(0)=I_{0}$, and $R(0)=R_{0}$, respectively. It is suggested to employ numerical methods to solve the ordinary differential equations when the analytic solutions are unavailable.

Step 3: Repeat Step 1 and Step 2 for 99 times.

Step 4: The solutions $S_{t}, I_{t}$, and $R_{t}$ have a 99-table,

\begin{tabular}{llll}
\hline 0.01 & 0.02 & $\cdots$ & 0.99 \\
\hline$S_{t}^{0.01}$ & $S_{t}^{0.02}$ & $\ldots$ & $S_{t}^{0.99}$ \\
$I_{t}^{0.01}$ & $I_{t}^{0.02}$ & $\cdots$ & $I_{t}^{0.99}$ \\
$R_{t}^{0.01}$ & $R_{t}^{0.02}$ & $\cdots$ & $R_{t}^{0.99}$ \\
\hline
\end{tabular}

This table gives an approximate inverse uncertainty distributions of $S_{t}, I_{t}$, and $R_{t}$ i.e., for any $\alpha=i / 100, i=1,2, \ldots, 99$, we can find $S_{t}^{\alpha}, I_{t}^{\alpha}, R_{t}^{\alpha}$ from the table such that

$$
\mathcal{M}\left\{S_{t} \geq S_{t}^{1-\alpha}, I_{t} \leq I_{t}^{\alpha}, R_{t} \leq R_{t}^{\alpha}, \forall t>0\right\}=\alpha .
$$

If $\alpha \neq i / 100, i=1,2, \ldots, 99$, then it is suggested to employ a numerical interpolation method to get approximate $S_{t}^{\alpha}, I_{t}^{\alpha}, R_{t}^{\alpha}$. The 99-method can be extended to 999-method if more precise uncertainty distributions for the uncertain system $(4 a-4 c)$ are needed.

\section{Calibration for numerical solutions}

We collect COVID-19 data of Hubei province from January 25 to April 23, 2020, which contains numbers of active cases and recovered cases. We put the whole data in 
Table 1 The numbers of active and recovered COVID-19 cases in Hubei from January 25 to April 23, 2020

\begin{tabular}{|c|c|c|c|c|c|c|c|c|}
\hline Data & I & $\mathrm{R}$ & Data & I & $\mathrm{R}$ & Data & I & $\mathrm{R}$ \\
\hline $2020 / 1 / 25$ & 958 & 42 & $2020 / 2 / 24$ & 41,660 & 20,912 & $2020 / 3 / 25$ & 2896 & 61,731 \\
\hline $2020 / 1 / 26$ & 1303 & 44 & $2020 / 2 / 25$ & 39,755 & 23,200 & $2020 / 3 / 26$ & 2526 & 62,098 \\
\hline $2020 / 1 / 27$ & 2567 & 47 & $2020 / 2 / 26$ & 36,829 & 26,403 & $2020 / 3 / 27$ & 2054 & 62,565 \\
\hline $2020 / 1 / 28$ & 3349 & 80 & $2020 / 2 / 27$ & 34,715 & 28,895 & $2020 / 3 / 28$ & 1733 & 62,882 \\
\hline $2020 / 1 / 29$ & 4334 & 90 & $2020 / 2 / 28$ & 32,959 & 31,187 & $2020 / 3 / 29$ & 1461 & 63,153 \\
\hline $2020 / 1 / 30$ & 5486 & 116 & $2020 / 2 / 29$ & 30,543 & 33,757 & $2020 / 3 / 30$ & 1283 & 63,326 \\
\hline $2020 / 1 / 31$ & 6738 & 166 & $2020 / 3 / 1$ & 28,216 & 36,167 & $2020 / 3 / 31$ & 1186 & 63,417 \\
\hline $2020 / 2 / 1$ & 8565 & 215 & $2020 / 3 / 2$ & 25,905 & 38,556 & $2020 / 4 / 1$ & 987 & 63,612 \\
\hline $2020 / 2 / 2$ & 10,532 & 295 & $2020 / 3 / 3$ & 24,085 & 40,479 & $2020 / 4 / 2$ & 834 & 63,762 \\
\hline $2020 / 2 / 3$ & 12,712 & 396 & $2020 / 3 / 4$ & 22,695 & 41,966 & $2020 / 4 / 3$ & 648 & 63,945 \\
\hline $2020 / 2 / 4$ & 15,679 & 520 & $2020 / 3 / 5$ & 21,239 & 43,468 & $2020 / 4 / 4$ & 577 & 64,014 \\
\hline $2020 / 2 / 5$ & 18,483 & 633 & $2020 / 3 / 6$ & 19,710 & 45,011 & $2020 / 4 / 5$ & 518 & 64,073 \\
\hline $2020 / 2 / 6$ & 20,677 & 817 & $2020 / 3 / 7$ & 18,303 & 46,433 & $2020 / 4 / 6$ & 448 & 64,142 \\
\hline 2020/2/7 & 23,139 & 1115 & $2020 / 3 / 8$ & 17,151 & 47,585 & $2020 / 4 / 7$ & 401 & 64,187 \\
\hline $2020 / 2 / 8$ & 24,881 & 1439 & $2020 / 3 / 9$ & 15,671 & 49,056 & $2020 / 4 / 8$ & 351 & 64,236 \\
\hline $2020 / 2 / 9$ & 26,965 & 1795 & $2020 / 3 / 10$ & 14,427 & 50,298 & $2020 / 4 / 9$ & 320 & 64,264 \\
\hline $2020 / 2 / 10$ & 28,532 & 2222 & $2020 / 3 / 11$ & 13,171 & 51,553 & $2020 / 4 / 10$ & 303 & 64,281 \\
\hline $2020 / 2 / 11$ & 29,659 & 2639 & $2020 / 3 / 12$ & 11,772 & 52,943 & $2020 / 4 / 11$ & 244 & 64,338 \\
\hline $2020 / 2 / 12$ & 43,455 & 3441 & $2020 / 3 / 13$ & 10,431 & 54,278 & $2020 / 4 / 12$ & 219 & 64,363 \\
\hline $2020 / 2 / 13$ & 46,495 & 4107.5 & $2020 / 3 / 14$ & 9605 & 55,094 & $2020 / 4 / 13$ & 179 & 64,402 \\
\hline $2020 / 2 / 14$ & 48,175 & 4774 & $2020 / 3 / 15$ & 8701 & 55,987 & $2020 / 4 / 14$ & 146 & 64,435 \\
\hline $2020 / 2 / 15$ & 49,030 & 5623 & $2020 / 3 / 16$ & 7795 & 56,883 & $2020 / 4 / 15$ & 122 & 63,494 \\
\hline $2020 / 2 / 16$ & 49,847 & 6639 & $2020 / 3 / 17$ & 6992 & 57,678 & $2020 / 4 / 16$ & 109 & 63,507 \\
\hline $2020 / 2 / 17$ & 50,338 & 7862 & $2020 / 3 / 18$ & 6287 & 58,381 & $2020 / 4 / 17$ & 105 & 63,511 \\
\hline $2020 / 2 / 18$ & 50,633 & 9128 & $2020 / 3 / 19$ & 5719 & 58,942 & $2020 / 4 / 18$ & 102 & 63,514 \\
\hline $2020 / 2 / 19$ & 49,665 & 10,337 & $2020 / 3 / 20$ & 5224 & 59,432 & $2020 / 4 / 19$ & 97 & 63,519 \\
\hline $2020 / 2 / 20$ & 47,627 & 13,577 & $2020 / 3 / 21$ & 4768 & 59,879 & $2020 / 4 / 20$ & 69 & 63,547 \\
\hline $2020 / 2 / 21$ & 46,439 & 15,299 & $2020 / 3 / 22$ & 4318 & 60,323 & $2020 / 4 / 21$ & 47 & 63,569 \\
\hline $2020 / 2 / 22$ & 44,604 & 16,738 & $2020 / 3 / 23$ & 3828 & 60,810 & $2020 / 4 / 22$ & 23 & 63,593 \\
\hline $2020 / 2 / 23$ & 43,369 & 18,854 & $2020 / 3 / 24$ & 3431 & 61,201 & $2020 / 4 / 23$ & 12 & 63,604 \\
\hline
\end{tabular}

Table 1. The population of Hubei is 59.27 million. Since the number of recovered cases on February 13 is missing, we use the average of two adjacent days to approximate.

Based on the growth of the data, we divide the data into two groups and estimate the parameters separately. Considering that Hubei changed the 'top leader' on February 13. Taking this day as a breakpoint, we divide the first 20 days as the first stage and the left days as the second stage. Employing the equations (20), we estimate the parameters. From the estimated results, we find $\beta_{2}=-1.6777 * 10^{-11}$ is a very small negative number and $\beta_{2} * N=-9.9514 * 10^{-4}$, which shows almost no contact rate. Such a tiny number could be ignored. This indicates that lockdown policy reaches 

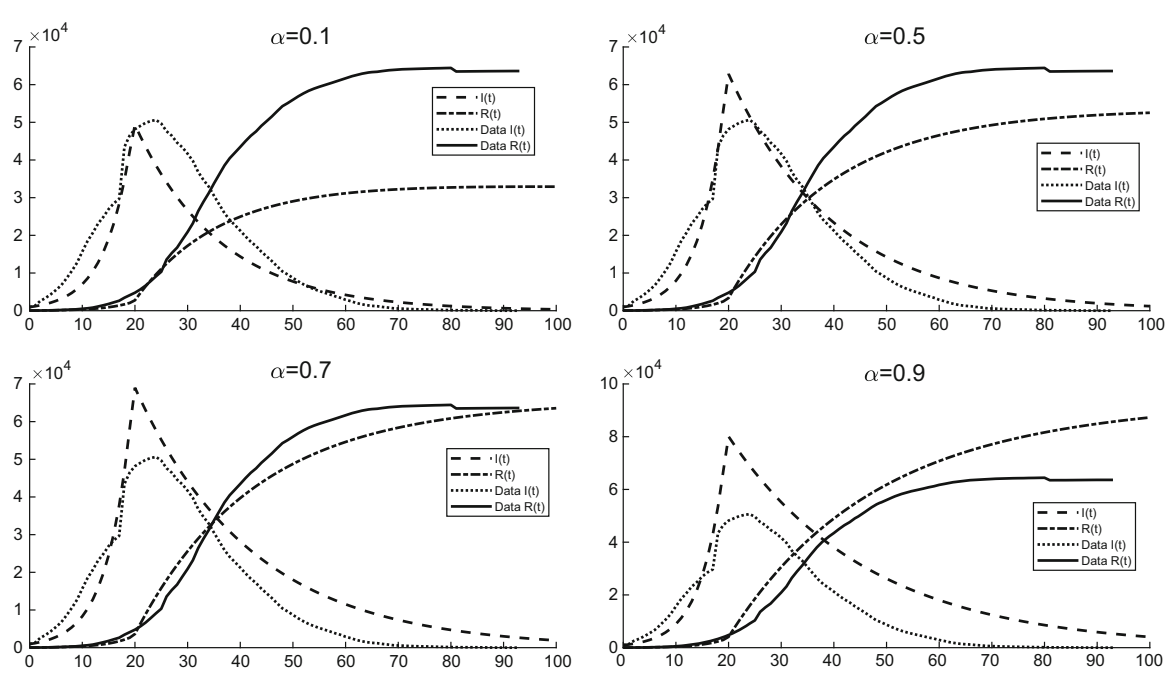

Fig. 1 The $\alpha$-path of the calibrated uncertain SIR model with $\alpha=0.1,0.5,0.7$ and 0.9

nearly $100 \%$ efficiency after replacing the 'top leader', which is consistent with the conclusions of the literature Li et al. (2020). Observing the numerical results, we find the estimated $\sigma_{I}$ and $\sigma_{R}$ are too large. We replace them with each divided by the total length of simulation dates. We put the estimate parameters bellow

$$
\begin{aligned}
\beta_{1} & =3.9015 * 10^{-9}, \quad \beta_{2}=-1.6777 * 10^{-11}, \\
\sigma_{I 1} & =1.0015 * 10^{-2}, \sigma_{I 2}=1.0065 * 10^{-2}, \\
\lambda_{1} & =9.7829 * 10^{-3}, \lambda_{2}=3.9456 * 10^{-2}, \\
\sigma_{R 1} & =1.2125 * 10^{-3}, \sigma_{R 2}=4.4196 * 10^{-4} .
\end{aligned}
$$

Plug the obtained number of each parameter into the uncertain SIR model, we have the follow first stage equations

$$
\begin{aligned}
\mathrm{d} S_{t} & =-3.9015 * 10^{-9} I_{t} S_{t} \mathrm{~d} t, S_{0}=59269000, \\
\mathrm{~d} I_{t} & =\left(3.9015 * 10^{-9} I_{t} S_{t}-1.9809 * 10^{-2} I_{t}\right) \mathrm{d} t+1.0015 * 10^{-2} I_{t} \mathrm{~d} C_{t}^{I}, I_{0}=958, \\
\mathrm{~d} R_{t} & =\left(9.7829 * 10^{-3} I_{t}-2.6027 * 10^{-5} R_{t}\right) \mathrm{d} t+1.2125 * 10^{-3} R_{t} \mathrm{~d} C_{t}^{R}, R_{0}=42,
\end{aligned}
$$

and the second stage equations

$$
\begin{aligned}
\mathrm{d} S_{t} & =1.6777 * 10^{-11} I_{t} S_{t} \mathrm{~d} t, S_{20}=59219397 \\
\mathrm{~d} I_{t} & =-1.6777 * 10^{-11} I_{t} S_{t}-4.9482 * 10^{-2} I_{t} \mathrm{~d} t+1.0065 * 10^{-2} I_{t} \mathrm{~d} C_{t}^{I}, \\
I_{20} & =46495 \\
\mathrm{~d} R_{t} & =\left(3.9456 * 10^{-2} I_{t}-\mu R_{t}\right) \mathrm{d} t+4.4196 * 10^{-4} R_{t} \mathrm{~d} C_{t}^{R}, R_{20}=4107.5 .
\end{aligned}
$$


We employ Runge-Kutta methods to calculate the numerical $\alpha$-path solutions. As shown in Fig. 1, all the observed data fall in the area nearly between the 0.1-path and the 0.9-path of the uncertain differential equation with the estimated parameter, so the estimates of $\beta, \lambda, \sigma_{I}$, and $\sigma_{R}$ are acceptable. Our model could be used not only to estimate the entire trend at the early stage of the pandemic, but also to update the parameters later to make more accurate estimates.

\section{Conclusions}

We build an uncertain SIR model with multiple diffusion sources via uncertain differential equations in this paper. In order to solve this high-dimensional uncertain differential equation system, $\alpha$-path-based approach is proposed. Based on it, we calculate the maximum uncertainty distributions and related expected values of the solutions. Furthermore, we employ the method of moments to estimate parameters and design a numerical algorithm to solve it. The uncertain SIR model is applied to describing the COVID-19 pandemic. Numerical calibrations are discussed with and without public data, respectively. Without data, the parameters follow the setting from literatures. Based on obtained uncertainty distributions of the solutions, we discuss potential demand for ICU beds and ventilators at the early stage of the pandemic. Using COVID-19 data in Hubei province, we estimate the parameters and solve the related $\alpha$-path solutions. Our results show that the parameter estimates are acceptable. The proposed high-dimensional $\alpha$-path-based approach opens up new possibilities in solving high-dimensional uncertain differential equations and new applications in other fields.

Acknowledgements This work is supported by National Natural Science Foundation of China Grant No. 61673225 and China Scholarship Council.

\section{References}

Bartlett, M. S. (1956). Deterministic and stochastic models for recurrent epidemics. In Proceedings of Third Berkeley Symposium on Math. Statistics and Probability, 4, 81-109.

Bendavid, E., Mulaney, B., Sood, N., et al. (2020). COVID-19 Antibody Seroprevalence in Santa Clara County. California: MedRxiv. https://doi.org/10.1101/2020.04.14.20062463.

Brauer, F., Castillo-Chavez, C., \& Feng, Z. (2019). Mathematical models in epidemiology. Berlin: Springer.

Chen, X., \& Liu, B. (2010). Existence and uniqueness theorem for uncertain differential equations. Fuzzy Optimization and Decision Making, 9(1), 69-81.

Chen, X., \& Gao, J. (2018). Two-factor term structure model with uncertain volatility risk. Soft Computing, 22(17), 5835-5841.

Fang, J., Li, Z., Yang, F., \& Zhou, M. (2018). Solution and $\alpha$-path of uncertain SIS epidemic model with standard incidence and demography. Journal of Intelligent \& Fuzzy Systems, 35(1), 927-935.

Hill, A., Levy, M., \& Xie, S., et al. (2020). Modeling COVID-19 spread versus healthcare capacity. 2020. Accessed, 3-25, https://alhill.shinyapps.io/COVID19seir/.

Iwata, K., \& Miyakoshi, C. (2020). A simulation on potential secondary spread of novel Coronavirus in an exported country using a stochastic epidemic SEIR model. Journal of Clinical Medicine, 9(4), 944.

Jia, J. S., Lu, X., Yuan, Y., et al. (2020). Population flow drives spatio-temporal distribution of COVID-19 in China. Nature. https://doi.org/10.1038/s41586-020-2284-y. 
Jia, L. F., \& Chen, W. (2020). Uncertain SEIAR model for COVID-19 cases in China. Fuzzy Optimization and Decision Making. https://doi.org/10.1007/s10700-020-09341-w.

Kermack, W. O., \& McKendrick, A. G. (1927). A contribution to the mathematical theory of epidemics. Proceedings of the Royal Society A, 115(772), 700-721.

Li, R., Pei, S., Chen, B., et al. (2020). Substantial undocumented infection facilitates the rapid dissemination of novel coronavirus (SARS-CoV-2). Science, 368(6490), 489-493.

Li, Z., Sheng, Y., Teng, Z., \& Miao, H. (2017). An uncertain differential equation for SIS epidemic model. Journal of Intelligent \& Fuzzy Systems, 33(4), 2317-2327.

Li, Z., Teng, Z., Hong, D., \& Shi, X. (2018). Comparison of three SIS epidemic models: Deterministic, stochastic and uncertain. Journal of Intelligent \& Fuzzy Systems, 35(5), 5785-5796.

Li, Z., \& Teng, Z. (2019). Analysis of uncertain SIS epidemic model with nonlinear incidence and demography. Fuzzy Optimization and Decision Making, 18(4), 475-491.

Liu, B. (2007). Uncertainty theory (2nd ed.). Berlin: Springer.

Liu, B. (2008). Fuzzy process, hybrid process and uncertain process. Journal of Uncertain Systems, 2(1), $3-16$.

Liu, B. (2009). Some research problems in uncertainty theory. Journal of Uncertain Systems, 3(1), 3-10.

Liu, B. (2010). Uncertainty Theory: A Branch of Mathematics for Modeling Human Uncertainty. Berlin: Springer-Verlag.

Liu, B. (2015). Uncertainty theory (4th ed.). Berlin: Springer.

Unwin, H. J. T., Mishra, S., Bradley, V. C., et al. (2020). Report 23: State-level tracking of COVID-19 in the United States. medRxiv. https://doi.org/10.1101/2020.07.13.20152355.

Yang, X., \& Shen, Y. (2015). Runge-Kutta method for solving uncertain differential equations. Journal of Uncertainty Analysis and Applications, 3(1), 1-12.

Yao, K., \& Chen, X. (2013). A Numerical method for solving uncertain differential equations. Journal of Intelligent \& Fuzzy Systems, 25(3), 825-832.

Yao, K., \& Liu, B. (2020). Parameter estimation in uncertain differential equations. Fuzzy Optimization and Decision Making, 19(1), 1-12.

Yao, K. (2015). Uncertain contour process and its application in stock model with floating interest rate. Fuzzy Optimization and Decision Making, 14(4), 399-424.

Publisher's Note Springer Nature remains neutral with regard to jurisdictional claims in published maps and institutional affiliations. 